\title{
The use of curved sheath technique to facilitate difficult internal carotid artery occlusions
}

\author{
Nitish Mittal BS, Sanjana Rao BS, Mohammad M Ansari MD
}

\begin{abstract}
Carotid artery occlusive disease or carotid artery stenosis, a widely prevalent disease, involves blockage of the arterial wall from atherosclerotic plaques. Due to the high prevalence of carotid artery stenosis, innovative approaches to improve outcomes are needed, particularly for more difficult and unusual cases. In this report, we present a particularly difficult case with a Type III aortic arch-right internal carotid artery stenosis. We describe a symptomatic 87-year-old man who presented to the cardiology clinic for evaluation. Computed tomography angiogram showed a $70 \%$ occlusion of the right internal carotid artery; the patient was considered high risk for carotid artery surgery. He underwent internal carotid artery stenting using the curved sheath technique with excellent results. This case demonstrates the importance of innovative new techniques in the development of medical treatment.
\end{abstract}

Keywords: Carotid artery stenting, curved sheath technique, type 3 aortic arch, right internal carotid artery stenosis

\section{INTRODUCTION}

Carotid artery stenosis (CAS) is a common disease, which presents with blockage of blood flow due to formation of atherosclerotic plaques. These plaques can either narrow the arterial lumen (stenosis) or completely block blood flow (occlusion). Carotid artery angioplasty with stenting, a minimally invasive procedure, has become a widely used procedure for carotid artery occlusive disease, especially in patients at high risk for endarterectomy. ${ }^{1}$ Symptomatic patients with $>50 \%$ internal carotid artery stenosis and asymptomatic patients with $>60 \%$ internal carotid artery stenosis undergo carotid endarterectomy as standard care. $^{2}$ In the past decade, carotid artery stenting has received more attention and has been used in multiple medical institutions.

Corresponding author: Mohammad Ansari

Contact Information: Mac.Ansari@ttuhsc.edu

DOI: 10.12746/swrccc.v9i37.787
Various types of aortic arches are present based on the vertical distance from the innominate artery to the top of the arch (Figure 1). ${ }^{3}$ The various measurements for assigning different types of aortic arches are: distance of $<1$ diameter of the left common carotid artery $(\mathrm{CCA})=$ Type $\mathrm{I}$ arch; between 1 and 2 CCA diameters = Type II arch; and $>2$ CCA diameters $=$ Type III arch. ${ }^{3}$ The selection of great vessels in the setting of a Type III arch can present a challenge and typically requires a reverse curved catheter. This makes it difficult for the interventional cardiologist to perform stenting due to the sharp angle created by the aortic arch.

\section{CASE}

An 87-year-old man presented to the cardiology clinic for elective carotid intervention. He had a previously diagnosed history of right frontal stroke, chronic kidney disease, hypertension (HTN), chronic systolic heart failure, carotid artery disease, and peripheral artery disease. The patient presented with shortness of breath; he denied chest pain, palpitations, 


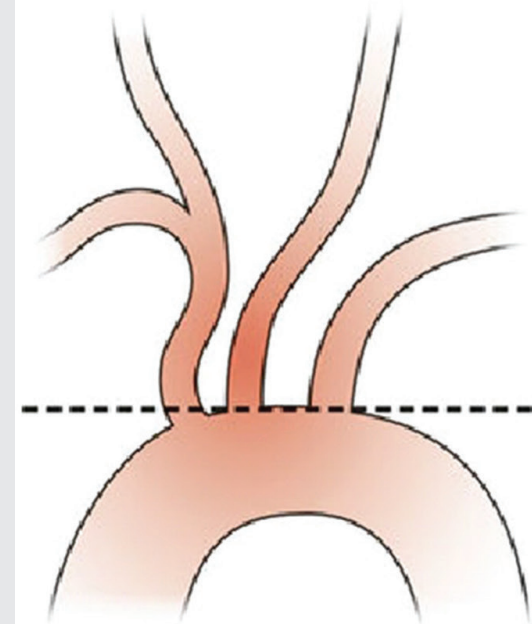

Type I

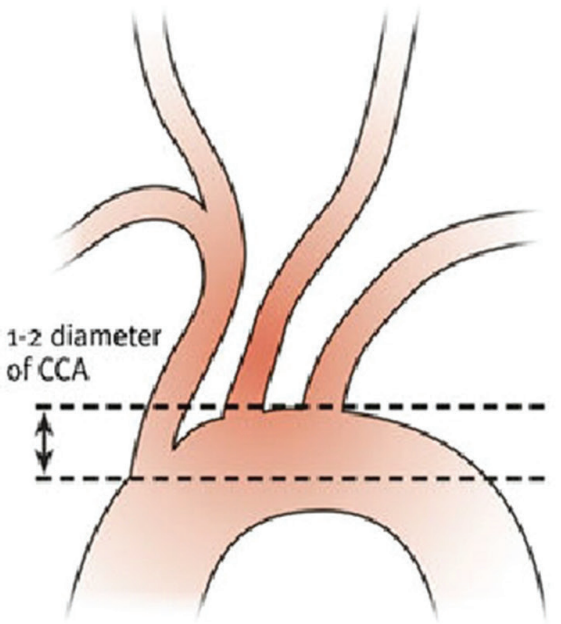

Type II

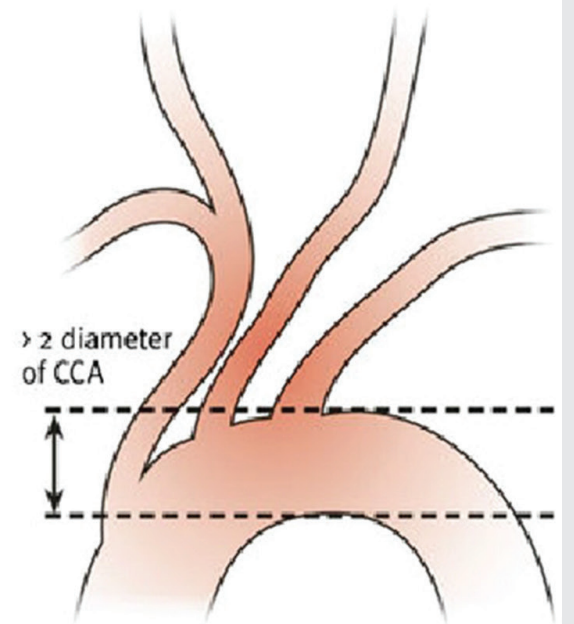

Type III

Figure 1. Different Types of Aortic Arch. Vertical distance from the innominate artery to the top of the arch.

and muscle or joint pain. A clinical diagnosis of internal carotid artery stenosis was made, and a carotid angiogram showed $70 \%$ occlusion of the right internal carotid artery. The stenosis had reduced the blood flow and increased the risk of emboli (Figure 2). Surgery was consulted and he was considered high risk for endarterectomy due to comorbidities and age. Hence, the patient was scheduled for internal carotid artery stenting in the catheterization laboratory.

The team proceeded with ultrasound-guided right common femoral artery access. The patient had a Type 3 aortic arch, which made advancement of the catheter difficult due to a sharp angle (Figure 3). Therefore, a curved sheath technique was used with $6-\mathrm{Fr}$ vascular sheath. The Cook $90 \mathrm{~cm}$ Shuttle Sheath was manually curved by the operator and then positioned in the right common carotid artery. This was followed by crossing the stenosis using a 0.014 Whisper wire. Eventually, a Medtronic Protege 8-6×40 mm carotid self-expanding stent was placed at the site of the lesion in the internal carotid artery after placing the SpiderFX embolic protection device, since other filters were unpassable, which significantly reduced the risk of emboli (Figure 4). After the procedure, the patient was transferred to the $\mathrm{CICU}$ in stable hemodynamic condition. Post-operatively, he developed bradycardia without hypotension transiently

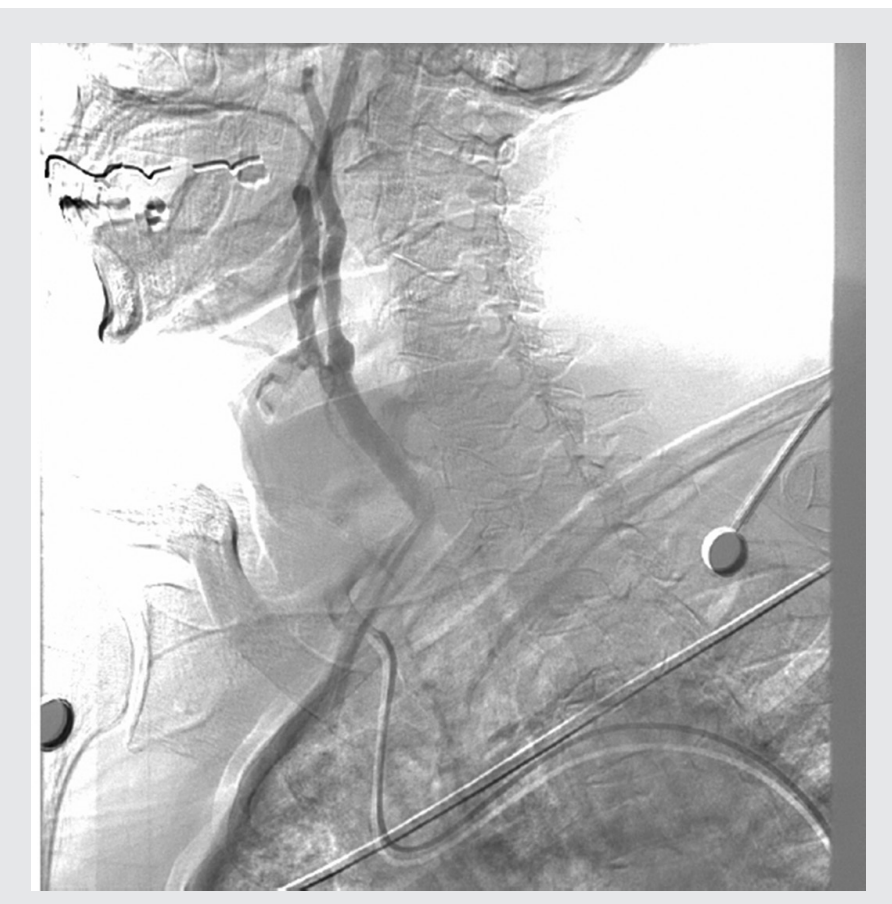

Figure 2. Carotid Artery Angiogram before Stenting. Right internal carotid artery stenosis reduces blood flow, which increases the risk of emboli.

requiring chronotropic support with a dopamine infusion, from which he was successfully weaned off in 


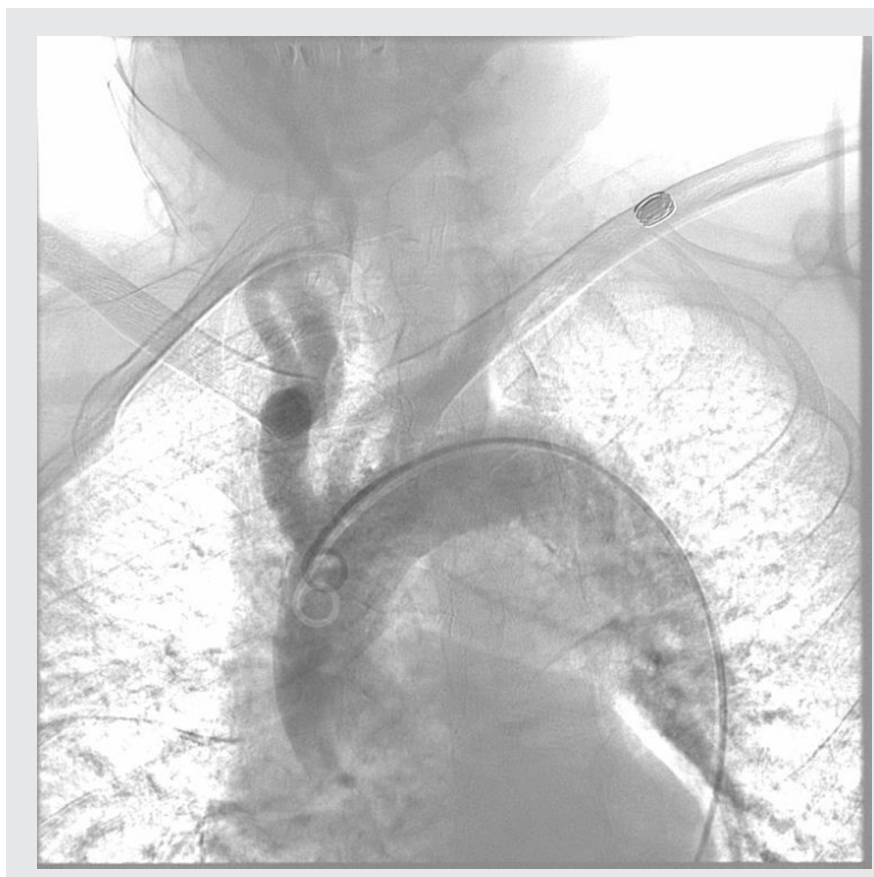

Figure 3. Type III Aortic Arch. Angiogram shows that the patient has Type III aortic arch $\geq 2$ diameter of common carotid artery.

a day. Reflexive bradycardia is a well described postprocedural phenomenon following internal carotid artery stenting.

\section{Discussion}

Carotid artery occlusive disease or carotid artery stenosis (CAS) is due to plaque (fat and cholesterol deposits) buildup in the arterial walls which disrupts blood flow. ${ }^{4}$ This blockage increases the risk of emboli and stroke, a medical emergency in which blood supply to the brain is interrupted. ${ }^{5}$ Stroke is the third major cause of death in the United States, and CAS is the underlying etiology in approximately $20-30 \%$ of cases. ${ }^{1}$ Carotid artery stenosis is more common in an older patients since atherosclerosis progresses slowly.

Several risk factors contribute to the development of carotid artery disease, including HTN, diabetes, hyperlipidemia, age, obesity, family history, physical inactivity, and tobacco use. ${ }^{5}$ In early stages, CAS often does not produce signs or symptoms, but later stages

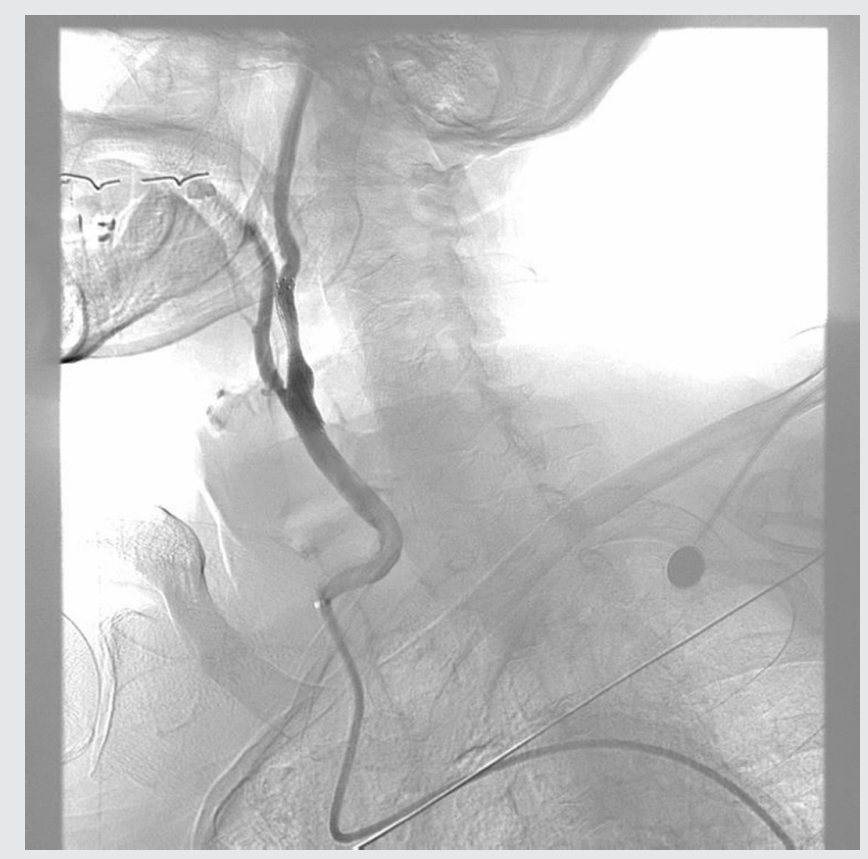

Figure 4. Carotid Artery Angiogram after Stenting. Stent placement increased blood flow slightly and significantly decreased the risk of emboli.

have a wide range of symptoms, including shortness of breath, numbness or weakness in extremities, headache, vision changes, and dysarthria. ${ }^{5}$ Our patient presented with similar symptoms and was evaluated for CAS based on the risk factors and presentation. The management of CAS involves computed tomography angiography followed by carotid artery stenting depending on the severity of stenosis. Postprocedure, patients are prescribed aspirin and clopidogrel for at least 6 months in combination. These medications help prevent the formation of blood clots where the stent was placed. ${ }^{4}$ This is followed by regular follow-up in the clinic to track the progress of the intervention.

The curved sheath technique has been used in challenging cases when other conventional medical treatment options were exhausted. For instance, catheterization of the coronary sinus is important in electrophysiology cases. In difficult cases where normal sheaths cannot be used for catheterization, a curved sheath is used to provide more stability and prevent 
catheter slide along with improving 3-D map quality. ${ }^{6}$ Our patient was diagnosed with Type III aortic arch by angiography. This arch creates a sharp angle and makes it difficult to enter the right internal carotid artery due to the high pressure blood flow from the heart. A radial technique was not used because at that time of the procedure, there were no FDA approved devices available for radial approach for the carotid stenting. In these situations, curved sheath techniques provide the stability needed to guide the catheter into the right internal carotid artery with minimal risk and complications. Post-procedure, there was a definite increase in the patient's blood flow, and the risk of emboli was significantly decreased.

This case illustrates the innovative use of the curved sheath technique to manage a challenging case of Type 3 aortic arch right internal carotid artery stenosis. Using the curved sheath technique circumvented the need for a carotid endarterectomy, thus mitigating risks in this particular case. This is a good example of how innovation and new techniques are integral in the advancement of medical treatment.

\section{Conclusion}

Carotid artery stenosis is becoming more common, especially in older patients with comorbidity. Computed tomography, Doppler studies, and digital subtraction fluoroscopic angiography are used as diagnostic tools if specific symptoms occur which raise suspicion for CAS. Treatment varies depending on the severity; medications are used for milder cases and stenting/ surgery for more severe symptomatic cases. Our case demonstrates the importance of the curved sheath technique in circumventing carotid endarterectomy and potential medical complications.
Article citation: Mittal N, Sanjana Rao S, Ansari MM. The use of curved sheath technique to facilitate difficult internal carotid artery occlusions. The Southwest Respiratory and Critical Care Chronicles 2021;9(37):74-77

From: Department of Internal Medicine, Texas Tech University Health Sciences Center, Lubbock, Texas

Submitted: $12 / 16 / 2021$

Accepted: 1/9/2021

Reviewer: Ankush Lahoti MD

Conflicts of interest: none

This work is licensed under a Creative Commons Attribution-ShareAlike 4.0 International License.

\section{REFERENCES}

1. Madhwal S, Rajagopal V, Bhatt DL, et al. Predictors of difficult carotid stenting as determined by aortic arch angiography. J Invasive Cardiol 2008 May;20(5):200-4.

2. Ahn SH, Prince EA, Dubel GJ. Carotid artery stenting: review of technique and update of recent literature. Semin Intervent Radiol 2013 Sep;30(3):288-96. doi: 10.1055/s-00331353482

3. Kotelis D, Bischoff MS, Jobst B, et al. Morphological risk factors of stroke during thoracic endovascular aortic repair. Langenbecks Arch Surg 2012 Dec;397(8):1267-73. doi: 10.1007/ s00423-012-0997

4. Cleveland Clinic. Carotid Artery Disease: Carotid Stenting. https://my.clevelandclinic.org/health/treatments/16850carotid-artery-disease-carotid-stenting. Accessed December 15, 2020.

5. Mayo Clinic. Carotid Artery Disease. https://www.mayoclinic. org/diseases-conditions/carotid-artery-disease/symptomscauses/syc-20360519. Accessed December 15, 2020.

6. Shandling AH, Rieders D, Edwards $M$. Use of a unique long pre-curved sheath to facilitate femoral placement of coronary sinus catheters. Indian Pacing Electrophysiol J 2009 Sep 1;9(5):247-50. 